\title{
Mucormycosis in a Diabetic Patient
}

\section{Jyotismita Thakuria ${ }^{1 *}$, Rajendragouda Patil ${ }^{2}$ and Udita Singh ${ }^{3}$}

${ }^{1}$ Post Graduation Student, Oral Medicine and Radiology, Kothiwal Dental College

and Research Centre, Moradabad, India

${ }^{2}$ Professor and Head, Oral medicine and Radiology, Kothiwal Dental College and

Research Centre, Moradabad, India

${ }^{3}$ Reader, Oral Medicine and Radiology, Kothiwal Dental College and Research

Centre, Moradabad, India

*Corresponding Author: Jyotismita Thakuria, Post Graduation Student, Oral Medicine

and Radiology, Kothiwal Dental College and Research Centre, Moradabad, India
Received: August 30, 2021

Published: September 25, 2021

(C) All rights are reserved by Jyotismita

Thakuria., et al.

\section{Abstract}

Though there have been an outbreak of mucormycosis infection in covid-19 patients, it was prevalent even before the pandemic. When the defense system of the human body is damaged or the concentration of pathogens reach an exceptionally high density, opportunistic infection such as mucormycosis may emerge. Mucormycosis is the most deadly and rapidly progressive form of fungal infection caused by a saprophytic fungus, mainly Rhizopus or Mucor. Mucorales gain entry to the susceptible host from the environment through skin contact with or without trauma, or via inhalation or ingestion of fungal spores. These routes result in rhino-orbitocerebral, pulmonary, gastrointestinal, or cutaneous/wound infections. Here we present a case of 42 year old diabetic patient who was diagnosed with mucormycosis causing extensive maxillary and alveolar necrosis before the Covid-19 pandemic.

Keywords: Fungal; Infection; Opportunistic; Mucormycosis; Necrosis; Black Fungus

\section{Abbreviations}

ESR: Erythrocyte Sedimentation Rate; WBC: White Blood Cell; IOPAR: Intra-Oral Periapical Radiograph; OPG: Orthopantomogram; CT: Computed Tomography

\section{Introduction}

Mucormycosis, also known as zygomycosis or phycomycosis is caused by the ubiquitous filamentous fungi of the Mucorales order of the class of Zygomycetes [1]. Mucorales can gain entry to a susceptible host through inhalation, ingestion of contaminated food, or through open wound. One of the characteristic features of mucormycosis is its angioinvasive property, resulting in host tissue infarction and tissue necrosis resulting from vasculature invasion by hyphae starting with a specific interaction with endothelial cells [2].
Mucormycosis especially affects immuno-compromised patients, though it may also affect immune-competent patients following a trauma or burn [3]. It is the third common fungal infection after candidiasis and aspergillosis. Up to $50 \%$ of patients presenting with mucormycosis are diabetics [4]. However, those occurring subsequent to tooth extraction are rare [5]. Diagnosis is difficult because of clinico-radiological similarities with invasive aspergillosis and early diagnosis and treatment is necessary because of its invasive property.

\section{Case Report}

A 42 year old male patient reported to the Department of Oral Medicine and Radiology with the chief complaint of escape of fluid from the nose after taking liquid and pain and ulceration in upper right premolar region since past 4 months. Pain was insidious in 
onset, dull, intermittent and non-radiating in nature. Patient was a known diabetic and was not on regular medication since 2 years. Dental history revealed that the patient had undergone extraction of a decayed tooth in upper right back region 4 months back followed by antibiotic prophylaxis for 5 days. Following extraction the socket never healed completely and later after a week, patient noticed an ulcer which gradually increased in size associated with pain at the extraction site. Over the past 4 months the patient was advised various antibiotics and analgesics by different dental practitioners and had very limited benefit. No deleterious habits or parafunctional habits was reported.

Extraorally, facial asymmetry was detected. A diffuse swelling was present in the right cheek region which was non tender. Intra oral examination revealed an infiltrating ulcer approximately $2 \times$ $3.5 \mathrm{~cm}^{2}$ with irregular borders adjacent to $1^{\text {st }}$ premolar region. The ulcer was covered by necrotic slough and the crestal alveolar bone was exposed and appeared yellowish white with no bleeding on probing. Surrounding mucosa appeared normal. On the mid palate region a small perforation was present approximately $5 \times 5 \mathrm{~mm}$ in size through which pus was draining (Figure 1). Maxillary right $1^{\text {st }}$ premolar was missing.

A provisional diagnosis of squamous cell carcinoma was made. The differential diagnosis of lethal midline granuloma and fungal infection were thought.

Routine blood investigation revealed raised ESR ( $40 \mathrm{~mm} / \mathrm{hour}$ ), decreased WBC count $3.5 \times 10^{3}$ micro litre and increased random blood glucose level of $360 \mathrm{mg} / 100 \mathrm{~mL}$.

An intra-oral periapical radiograph (IOPAR) of the 14 region was taken with a gutta percha cone inserted into the socket. IOPAR showed an abnormal communication between the oral cavity and maxillary sinus suggestive of oro-antral fistula.

The patient was advised an OPG which showed discontinuity of the floor of the right maxillary sinus.

The patient was then subjected to computed tomography (CT) scan in order to study the extent and location of the lesion. Sectional views of CT scan showed thickening of right maxillary antrum lining, with destruction and perforation of anterior maxillary wall.
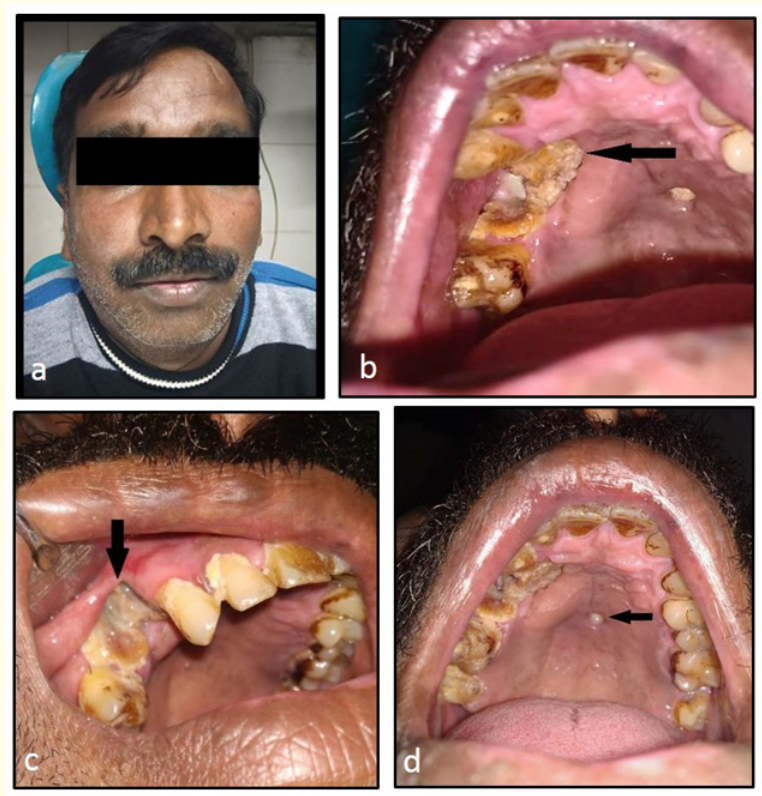

Figure 1a-1d: Figure 1a: Extra-oral image showing a diffuse swelling in the right cheek region. Figure $1 \mathrm{~b}$ and 1c: Intra oral picture showing infiltrating ulcer with irregular borders adjacent to $1^{\text {st }}$ premolar region in palatal and buccal aspect respectively. Figure 1d: Perforation on the mid palate region.

Three dimensional reformatted CT image showed destruction of right anterior maxillary wall extending antero-posteriorly from the lateral nasal wall to the zygomatic bone and supero-inferiorly from the crest of the maxillary alveolar bone to orbital floor. Presence of bony sequestrum was noted (Figure 2).

Radiological diagnosis of Osteomyelitis causing oro antral fistula of right side of maxilla was made.

Gingival and bone incision biopsies were taken for histopathological study. The microscopic evaluation showed loose stromal tissue. The stroma showed loose collagen fibres, very few bony trabeculae, along with mixed inflammatory cell infiltrate comprising of neutrophils, lymphocytes and plasma cells. Areas of haemorrhage and necrosis were evident. Numerous broad and aseptate fungal hyphae with irregular branching was seen. The features 


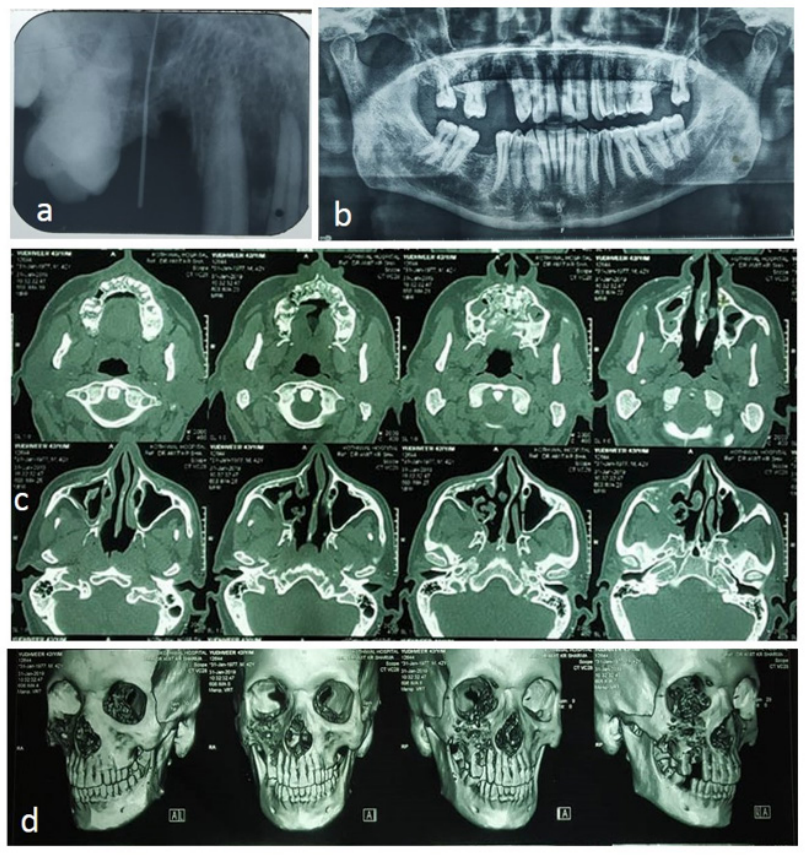

Figure 2a-2d: Figure 2a: IOPAR showing an abnormal communication between the oral cavity and maxillary sinus. Figure 2b: OPG which showing discontinuity of the floor of the right maxillary sinus. Figure 2c: CT scan showing thickening of right maxillary antrum lining, with destruction and perforation

of anterior maxillary wall. Figure 2d: Three dimensional reformatted CT image showed destruction of right anterior maxillary wall with presence of bony sequestrum.

were suggestive of mucormycosis. A final diagnosis of Maxillary osteonecrosis secondary to mucormycosis was made.

The patient was later refered to the Department of Oral Surgery where the patient underwent partial maxillectomy.

The specimen was creamish white, non-vital, measured about $5 \times 3 \mathrm{~cm}$ with rough surface. Patient was then put on intravenous antifungal therapy, Amphotericin B; $0.8 \mathrm{mg} / \mathrm{kg} /$ day for 3 weeks. Periodic monitoring of blood sugar was done during the therapy. The patient showed uneventful recovery and was kept on regular recall visits (Figure 3).

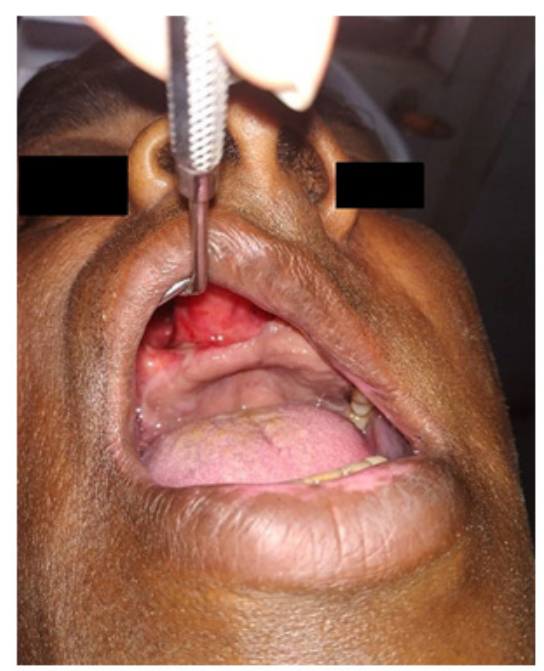

Figure 3: Recovery 2 weeks after surgery.

Patient was rehabilitated with an obturator and was being followed up for 6 months and had no further complications.

\section{Discussion}

Mucormycosis is an invasive fungal infection first described by Paulltauf in 1885 [6]. Early diagnosis and distinction from invasive aspergillosis is of utmost importance, as the underlying conditions and clinical presentation are often similar. In our case, diagnosis was prompt and mainly done through the imaging studies and positive histological findings. The recommended guidelines for mucormycosis which are antifungal treatment, surgical debridement, and correction of risk factors were followed. Amphotericin B (Amb) and its lipid formulations and posaconazole were the only antifungal drugs available with in vitro activity against mucorales [7]. Adjunctive therapy with recombinant cytokines, hyperbaric oxygen, and/ or granulocyte transfusions can be considered too. Our patient was administered Amphotericin B intra-venously after surgery. There was complete resolution of clinical signs and symptoms of infection as well as resolution of radiographic signs of mucormycosis after treatment. Nephrotoxicity is a limiting adverse side effect of Amphotericin B, however in our case the patient's renal function tests were under normal limits. Underlying immunosuppression 
which is diabetes in our case was regularly monitored and kept under control with subsequent follow ups.

\section{Conclusion}

Early diagnosis, reversal of underlying predisposing risk factors, surgical debridement where applicable and prompt antifungal therapy are the steps for the successful treatment of mucormycosis. Though incidence of mucormycosis secondary to tooth extraction is extremely low, however when it occurs, may cause significant morbidity and mortality. Hence dental professionals must be aware of the possibility of this serious and fatal complication, so as to avoid unfavorable outcome in clinical practice.

\section{Conflict of Interest}

None.

\section{Bibliography}

1. Lehrer RI., et al. "Mucormycosis". Annals of Internal Medicine 93 (1980): 93-108.

2. Nilesh K and Vande AV. "Mucormycosis of maxilla following tooth extraction in immunocompetent patients: Reports and review". Journal of Clinical and Experimental Dentistry 10.3 (2018): e300-305.

3. Hosseini SM and Borghei P. "Rhinocerebral mucormycosis: pathways of spread". European Archives of Oto-Rhino-Laryngology 262 (2005): 932-938.

4. Roden MM., et al. "Epidemiology and outcome of zygomycosis: a review of 929 reported cases". Clinical Infectious Diseases 41.5 (2005): 634-653.

5. Kim J., et al. "A Fatal Outcome From Rhinocerebral Mucormycosis After Dental Extractions: A Case Report". Journal of Oral and Maxillofacial Surgery 59 (2001): 693-697.

6. Jones AC., et al. "Mucormycosis of the oral cavity". Oral Surgery, Oral Medicine, Oral Pathology and Oral Radiology 75 (1993): 455-460.

7. Cornely OA., et al. "ESCMID and ECMM joint clinical guidelines for the diagnosis and management of mucormycosis 2013". Clinical Microbiology and Infection 20.3 (2014): 5-26.

Volume 5 Issue 10 October 2021

(C) All rights are reserved by Jyotismita Thakuria., et al. 